\title{
Nurse-led management of heart failure outpatients
}

\author{
Urška Osredkar* \\ University Medical Centre \\ Ljubljana, Ljubljana, Slovenia
}

KEYWORDS: management, heart failure, outpatients.

CITATION: Cardiol Croat. 2016;11(10-11):577. | DOI: http://dx.doi.org/10.15836/ccar2016.577

*ADDRESS FOR CORRESPONDENCE: Urška Osredkar, Univerzitetni klinični center Ljubljana, Zaloška cesta 2, 1000 Ljubljana, Slovenia. / Phone: +386-40577358

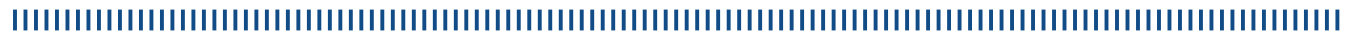

Since its establishment in 1999, the Heart Failure Outpatient Clinic (at Department of Vascular Diseases, University Medical Center in Ljubljana, Slovenia) supplemented cardiovascular diagnostic assessment, therapeutic management and follow-up with a structured and comprehensive nurse-led heart failure education program aimed at empowering outpatients with chronic heart failure.

In 2012, a pilot program of nurse-led heart failure management (including education, but also follow-up and heart failure medication optimization with uptitration of baseline therapy and adjustment of diuretic dosage) was started. The pilot program of nurse-lead heart failure management consisted of at least 4 visits every $2-3$ weeks and included 100 patients (mean age $80 \pm 6$ years, $39 \%$ female); the program accomplished an increase in patients taking optimal heart failure medication (i.e. $38 \%$ on maximal ACE inhibitor dose as compared to $18 \%$ in the usual care group, $\mathrm{p}=0.002$ ) and a decrease in patients with congestion/weight gain (12 vs. $42 \%$ in the usual care group, $\mathrm{p}<0.001)$.

From 2015, the nurse-led heart failure management program was implemented as the standard of care at the Heart Failure Outpatient Clinic (3 days/week - once a week for first visits/intake and twice a week for follow-up visits). At first visit/intake, outpatients referred for evaluation of suspected heart failure undergo thorough cardiovascular examination (including cardiologist assessment, echocardiography, blood analysis, 6-minute walk test) and if heart failure is confirmed, appropriate therapy is initiated by the managing cardiologist and education is carried out by a dedicated nurse. A structured and comprehensive education program addresses heart failure natural history, signs/symptoms, precipitating factors and signs of impending worsening, management, diuretic therapy, lifestyle intervention etc. Also, follow-up appointments with managing nurse are arranged and patients are followed at 2-3 weeks interval, with basic check-up (symptoms, adherence to medication, blood pressure, heart rate, body weight) and therapy dosage optimization (uptitration of ACE inhibitors/ARBs, beta blockers, MRAs and sacubitril/valsartan, and dose adjustment of diuretic therapy) under supervision and in consultation with managing cardiologist.

Nurse-led heart failure management programs improve outpatients follow-up yielding to better medication adherence and better optimization of both, diuretic therapy and lifesaving heart failure medication (thus possibly decreasing hospitalization rates and even mortality). ${ }^{1}$ Also, continuous education through frequent follow-up visits enables better outpatient empowerment and possibly improves quality of life.
RECEIVED:

October 3, 2016

ACCEPTED:

October 12, 2016

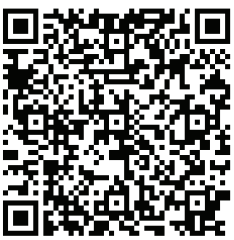

LITERATURE IIIIIIIIIIIIIIIIIIIIIIIIIIIIIIIIIIIIIIIIIIIIIIIIIIIIIIIIIIIIIIIIIIIIIIIIIIIIIIIIIIIIIIIIIIIIIIIII

1. Young JB. Evolution of heart failure management: Miles to go. Cleve Clin J Med. 2016;83(10):766-8. DOI: http://dx.doi.org/10.3949/ccjm.83a.16043.
Cardiologia Croatica $\square$ 2016;11(10-11):577. 